\title{
Solving the Stochastic Growth Model by Using Quadrature Methods and Value-Function Iterations
}

\author{
George Tauchen \\ Department of Economics, Duke University, Durham, NC 27706
}

\begin{abstract}
This article presents a solution algorithm for the capital growth model. The algorithm uses valuefunction iterations on a discrete state space. The quadrature method is used to set the grid for the exogenous process, and a simple equispaced scheme in logarithms is used to set the grid for the endogenous capital process. The algorithm can produce a solution to within four-digit accuracy using a state space composed of 1,800 points in total.
\end{abstract}

KEY WORDS: Discrete methods; Dynamic programming; Numerical approximations.

\section{INTRODUCTION}

This article describes a discrete state-space solution algorithm for the stochastic growth model set forth by Taylor and Uhlig (1990). In previous work (Tauchen 1985a,b, 1987), I used discrete methods to approximate a version of Lucas's asset-pricing model. For that model, discrete methods have many attractive features, the foremost being computational simplicity. In the discrete case, solution of the asset-pricing equations reduces to the essentially trivial task of inverting a matrix. This task contrasts sharply with the task of finding the exact solution of Lucas's model, which entails solving a Fredholm equation of the second kind and is not at all straightforward.

The main complicating factor of the stochastic growth model is the presence of an endogenous state variable (the capital stock). An endogenous state variable is an endogenous variable whose solution function depends on the infinite past of the exogenous driving process. Because of the presence of the endogenous state variable, the methods of my earlier articles have to be adapted and extended somewhat to work with this growth model. In brief, the strategy adopted in this article is to construct the discrete state space by using a simple equispaced grid (in logarithms) for the capital stock in conjunction with a quadrature-based grid for the exogenous technology shock that drives the model. The discretized model is then solved using value-function iterations.

The algorithm, which is coded in GAUSS and run on a Compaq 386-25, appears to be quite successful. When applied to a slightly different version of the problem in which the exact solution is known, the algorithm can approximate the exact solution with four-digit accuracy and with a computational time of about $40-45$ minutes.

Even though the algorithm approximates the decision rule closely, it still might appear to do poorly on criteria that test for statistical violations of orthogonality conditions implied by the Euler equation. The reason is that a value-function approach does not explicitly impose the Euler equation on the discrete model. Thus, for a fixed discretization, tests of orthogonality conditions will be rejected with probability tending to 1 as the length of a simulated realization increases. Though value-function iterations are used here, quadrature methods are sufficiently flexible that discretized models can also be solved by a Euler-equation approach. In my earlier work with Lucas's simple asset-pricing model, the Euler equation was imposed explicitly for the discrete model. Moreover, Hussey (1989) combined the quadrature approach and Coleman's (1989) Eulerequation method to obtain very good approximations to a factor-demand model with nonquadratic adjustment costs.

\section{THE STOCHASTIC GROWTH MODEL}

Since the model is set forth in detail by Taylor and Uhlig (1990), only a brief description is given here. The representative agent's problem is

$$
\begin{aligned}
\max E_{t} & {\left[\sum_{j=0}^{\infty} \beta^{j} u\left(c_{t+j}\right)\right] } \\
& \text { subject to } c_{t}+k_{t}-k_{t-1}=f\left(k_{t-1}, \theta_{t}\right),
\end{aligned}
$$

with $k_{t-1}$ and $\theta_{t}$ given. The functional forms are

$$
\begin{aligned}
u(c) & =\frac{1}{(1-\tau)} c^{(1-\tau)} \\
f(k, \theta) & =\theta k^{\alpha} \\
\log \left(\theta_{t}\right) & =\rho \log \left(\theta_{t-1}\right)+\varepsilon_{t}, \quad|\rho|<1, \quad \varepsilon_{t} \text { iid } N\left(0, \sigma_{\varepsilon}^{2}\right) .
\end{aligned}
$$


The Bellman equation is

$$
\begin{aligned}
v\left(k_{t-1}, \theta_{t}\right)=\max _{k} & \left\{u \left(f\left(k_{t-1}, \theta_{t}\right)\right.\right. \\
& \left.\left.+k_{t-1}-k\right)+\beta E_{t}\left[v\left(k, \theta_{t+1}\right)\right]\right\},
\end{aligned}
$$

where $v\left(k_{t-1}, \theta_{t}\right)$ is the optimized value of the objective function.

The case $\tau=0$ (linear utility) proves to be useful in calibrating the discrete state space. As can easily be checked, when $\tau=0$, the Euler equation for this model admits an explicit solution with log-linear decision rule

$$
\begin{aligned}
\log \left(k_{t}\right) & =(1-\alpha)^{-1} \\
\times & \left\{\log (\alpha \beta /(1-\beta))+(1 / 2) \sigma_{\varepsilon}^{2}+\rho \log \left(\theta_{t}\right)\right\} .
\end{aligned}
$$

In this case $\log \left(k_{t}\right)$ is normally distributed with unconditional moments

$$
\begin{aligned}
\mu_{1 k} & =E\left[\log \left(k_{t}\right)\right] \\
& =(1-\alpha)^{-1}\left\{\log (\alpha \beta /(1-\beta))+(1 / 2) \sigma_{\varepsilon}^{2}\right\} \\
\sigma_{1 k}^{2} & =\operatorname{var}\left[\log \left(k_{t}\right)\right]=\rho^{2} \sigma_{\varepsilon}^{2} /\left[\left(1-\rho^{2}\right)(1-\alpha)^{2}\right] .
\end{aligned}
$$

\section{DISCRETE APPROXIMATION}

The discrete state space for the capital stock and the technology shock is of the form $\{(k(i), \theta(j))\}$, where $i=1,2, \ldots, N_{1}$ and $j=1,2, \ldots, N_{2}$; the determination of the $k(i)$ and $\theta(j)$ is described more fully in Section 4 . Thus at any point in time the state vector of this economy $\left(k_{t-1}, \theta_{t}\right)$ assumes one of the $N_{1} \cdot N_{2}$ values of $(k(i), \theta(j))$. The law of motion of the discrete technology shock $\theta_{t}$ is the Markov chain $\pi\left(j, j^{\prime}\right)=\operatorname{Pr}\left[\theta_{t}=\right.$ $\left.\theta\left(j^{\prime}\right) \mid \theta_{t-1}=\theta(j)\right]\left(1 \leq j, j^{\prime} \leq N_{2}\right)$.

The discrete analog of the Bellman equation (3) is

$$
\begin{array}{r}
v(i, j)=\max _{i^{\prime}}\left\{u\left(f(k(i), \theta(j))+k(i)-k\left(i^{\prime}\right)\right)\right. \\
\left.+\beta \sum_{j^{\prime}=1}^{N_{2}} \pi\left(j, j^{\prime}\right) v\left(i^{\prime}, j^{\prime}\right)\right\},
\end{array}
$$

where $v(i, j)$ denotes the optimized value of the objective function given that the state of system at time $t$ is $(k(i), \theta(j))$. If a unique solution to the Bellman equation exists, then it defines a decision rule $i^{\prime}(i, j)$ giving the optimal choice, $k\left(i^{\prime}(i, j)\right)$ for each state $(i, j)$, and consumption is determined residually as $c(i, j)=$ $f(k(i), \theta(j))+k(i)-k\left(i^{\prime}(i, j)\right)$.

\section{IMPLEMENTATION}

Given values for the five parameters, $\beta, \tau, \alpha, \rho$, and $\sigma_{\varepsilon}$, the calibration and solution of the discrete problem proceeds as follows.

First, the quadrature method of Tauchen (1987) is used to calibrate the Markov chain for the technology shock. In this method, the discrete values of the Markov chain and the transition probabilities are constructed from a quadrature rule for integration against $\psi\left(\theta_{t} \mid 0\right)$, where $\psi\left(\theta_{t} \mid \theta_{t-1}\right)$ is the one-step density of the continuous-valued process $\left\{\theta_{t}\right\}$. Since the underlying (autoregressive) AR(1) model for $\left\{\theta_{t}\right\}$ is Gaussian, a GaussHermite rule is appropriate.

The next step is to define the discrete state space for the capital stock-that is, to lay out the grid composed of $N_{1}$ values $\{k(i)\}$. The quadrature method is not directly suited for this task, because it works by taking a fixed and given law of motion and calibrating a discrete approximation. In this problem, however, $k_{t}$ is an endogenous state variable whose law motion constitutes part of the solution. The strategy adopted here is to lay out an equispaced grid in $\log \left(k_{t}\right)$, where the range of the grid is determined by the analytical solution (4) for the case with linear utility. The $k(i)$ are given by

$$
\begin{aligned}
\log (k(i))=\mu_{1 k}-4.0 \sigma_{1 k}+\frac{8(i-1)}{N_{1}-1} \sigma_{1 k}, & \\
& \quad i=1,2, \ldots, N_{1},
\end{aligned}
$$

where $\mu_{1 k}$ and $\sigma_{1 k}$ are defined in (5). This is an equispaced grid extending for four standard errors on either side of the unconditional mean of $\log \left(k_{t}\right)$, with the unconditional mean and standard errors of $\log \left(k_{t}\right)$ being those of the special case $\tau=0$ (linear utility). The effects of using the linear-utility model to set the grid are small. In the deterministic version of this problem, the steady-state capital stock does not depend on $\tau$, indicating that $\tau$ does not exert too strong an influence on the location of the central part of the probability distribution of the capital stock.

Given $\{k(i)\},\{\theta(j)\}$, and $\left\{\pi\left(j, j^{\prime}\right)\right\}$ calculated in this manner the decision rule $i^{\prime}(i, j)$ is computed by iterating on the value function in the Bellman equation. Specifically, with $v_{r}(i, j)$ denoting the value function at the $r$ th iteration, then $v_{r+1}(i, j)$ is obtained by

$$
\begin{array}{r}
v_{r+1}(i, j)=\max _{i^{\prime}}\left\{u\left(f(k(i), \theta(j))+k(i)-k\left(i^{\prime}\right)\right)\right. \\
\left.+\beta \sum_{j^{\prime}=1}^{N_{2}} \pi\left(j, j^{\prime}\right) v_{r}\left(i^{\prime}, j^{\prime}\right)\right\} .
\end{array}
$$

These iterations are repeated until the condition $\left[\max _{i, j}\left\{\left|v_{r+1}(i, j)-v_{r}(i, j)\right|\right\}\right] / \min _{i, j}\left\{v_{r}(i, j)\right\}<$ toler is met, where toler is the relative convergence criterion. Experience suggested that using deterministic problems, where $\theta_{t}=\theta(j)$ for all $t$, to compute an initial value function $v_{0}(i, j)$ provided good start values and the convergence was reasonably rapid.

\section{SETTINGS OF TUNING PARAMETERS AND ACCURACY CHECKS}

The details are as follows: In each case, a 20-point quadrature rule was used to calibrate a Markov-chain model for $\left\{\theta_{t}\right\}$ and hence $N_{2}=20$ throughout. Moreover, $N_{1}=90$ points were used to lay out the grid (7) for the capital stock. Given that $N_{1}=90$ and $N_{2}=20$, 
then there are 1,800 states in total for the discrete system. Finally, toler $=.001$ was used as the relative convergence criterion. These settings yield four-digit accuracy.

The algorithm was coded in Gauss $1.49 \mathrm{~b}$ from Aptech Systems, which employs double precision arithmetic. It was executed on a Compaq 386-25 running MS-DOS, version 3.3, from Compaq Corporation. The central processing unit chip and the 80387 math coprocessor run at 25 megahertz. Speed benchmarks for this equipment are 5.5 million instructions per second and .25 Linpack double precision million floating point operations per second (Rinko-Gay 1989). The total time required to solve case 1 of Taylor and Uhlig $(1990)$ and to generate a simulated realization of length 2,000 was 46 minutes and 8.2 seconds.

Table 3 of the Taylor-Uhlig article contains solutions for this algorithm evaluated over the $5 \times 5$ grid of $k$ 's and $\theta$ 's that were specified for comparison of different solution algorithms. The grids used to display and compare solutions were chosen independently of the solution method described in this article and thus in no case will the $k(i)$ 's and $\theta(j)$ 's of the solution algorithm lie exactly on the corresponding grid for that case. Thus a locally linear interpolation scheme is used to interpolate a value $k^{\prime}(k, \theta)$ from the values $k^{\prime}(i, j)=k\left(i^{\prime}(i, j)\right)$ delivered by the solution algorithm. The linear interpolation scheme appears to work fine, except when $k$ or $\theta$ is near the edge or outside the range of points $(k(i)$, $\theta(j))$ of the solution algorithm.

To assess numerical accuracy, the preceding procedure was applied to a slightly different version of the problem (Sargent 1987, p. 36) of which the exact solution is known. This problem has $u(c)=\log (c)$ and $100 \%$ depreciation.

Table 1, which is representative of several cases studied, shows the exact and approximate decision rules for capital when the algorithm is applied to Sargent's model. The parameter settings are those of case 9 described in the Taylor-Uhlig article, except that $\tau$ is set to .99999 . The algorithm is only off by one digit or so in the fourth decimal place. This is essentially four-digit accuracy, which is the conventional standard for numerical work. A similar degree of accuracy was obtained for the consumption-decision rule.
Table 1. Decision Rules for Capital When the Algorithm Is Applied to Sargent's Model

\begin{tabular}{lccccc}
\hline \hline & \multicolumn{5}{c}{$k$} \\
\cline { 2 - 6 }$\theta$ & .90 & .95 & 1.00 & 1.05 & 1.10 \\
\hline \multicolumn{5}{c}{ Exact } \\
.14 & .1475 & .1557 & .1639 & .1720 & .1802 \\
.16 & .1541 & .1627 & .1712 & .1798 & .1884 \\
.18 & .1602 & .1691 & .1780 & .1869 & .1958 \\
.20 & .1659 & .1751 & .1843 & .1935 & .2028 \\
.22 & .1712 & .1807 & .1902 & .1997 & .2092 \\
& \multicolumn{5}{c}{ Approximate } \\
.14 & .1475 & .1556 & .1640 & .1721 & .1801 \\
.16 & .1540 & .1627 & .1714 & .1799 & .1883 \\
.18 & .1602 & .1689 & .1778 & .1869 & .1960 \\
.20 & .1660 & .1751 & .1842 & .1936 & .2026 \\
.22 & .1713 & .1807 & .1901 & .1997 & .2094 \\
\hline
\end{tabular}

\section{ACKNOWLEDGMENTS}

This work was supported by National Science Foundation Grant SES-8520244. I thank Lars Hansen for helpful discussions in the early stages of the work and Bob Hussey for many helpful comments and expert research assistance.

\section{[Received December 1988.] \\ REFERENCES}

Coleman, W. J. (1989), "An Algorithm to Solve Dynamic Models," International Finance Discussion Paper 351, Board of Governors of the Federal Reserve, Washington, DC.

Hussey, R. (1989), "Seminonparametric Modeling of Aggregate Employment Dynamics," unpublished Ph.D. dissertation, Duke University, Dept. of Economics.

Rinko-Gay, W. L. (1989), "Three 386 Architectures Run Their Course," MIPS, June, 69-76.

Sargent, T. J. (1987), Dynamic Macroeconomic Theory, Cambridge, MA: Harvard University Press.

Tauchen, G. (1985a), "Statistical Properties of GMM Estimators of Structural Parameters Obtained From Financial Market Data," Journal of Business and Economic Statistics, 4, 397-415.

(1985b), "Finite State Markov Chain Approximations to Univariate and Vector Autoregressions," Economic Letters, 20, 177181.

_ (1987), "Quadrature-Based Methods for Obtaining Approximate Solutions to Nonlinear Asset Pricing Models," unpublished manuscript, Duke University, Dept. of Economics.

$\rightarrow$ Taylor, J. B., and Uhlig, H. (1990), "Solving Nonlinear Stochastic Growth Models: A Comparison of Alternative Solution Methods," Journal of Business and Economic Statistics, 8, 1-17. 